\title{
An audit of retinal lymphoma treatment at the University of California San Francisco
}

\author{
Bertil Damato $^{1,2} \cdot$ Greg J. Bever $^{1} \cdot$ Dan J. Kim ${ }^{1} \cdot$ Armin R. Afshar $^{1} \cdot$ James L. Rubenstein $^{3}$
}

Received: 3 February 2019 / Revised: 11 June 2019 / Accepted: 14 June 2019 / Published online: 29 July 2019

(c) The Author(s), under exclusive licence to The Royal College of Ophthalmologists 2019

\begin{abstract}
Background/objectives To evaluate retinal lymphoma treatment at the University of California San Francisco.

Subjects/methods Prospective observational audit. Patients were treated systemically, usually with: methotrexate, temozolomide, and rituximab induction; etoposide consolidation; and maintenance with lenalidomide or another immunomodulatory agent. Persistent disease was treated initially by ocular radiotherapy or intravitreal melphalan and latterly by vitrectomy.

Results The cohort comprised eight females and two males. The median age was 58 years (range, 38-73). Ocular manifestations were initially unilateral in four patients. Vitreous and subretinal infiltrates were initially present in 16 and 12 eyes, respectively, with three eyes having vision of 20/200 or worse. Four patients had a history of central nervous system (CNS) lymphoma. The median ophthalmic follow-up was 37 months (range, 8-56). Diagnostic and therapeutic vitrectomy were performed in 10 and 2 eyes, respectively. All patients had systemic chemotherapy and eight received maintenance immunotherapy. Four patients underwent ocular radiotherapy, bilaterally in two. One patient received bilateral intravitreal melphalan injections. Two eyes of four patients developed lymphoma during the study and two patients developed CNS disease. At study close, subretinal deposits were subtle in nine eyes and more prominent in two, whereas vitreous infiltrates were minimal in nine eyes, mild in one and moderate in one. The latest visual acuity was significantly worse than at presentation in two eyes and better in two. All patients were alive with no active CNS disease.

Conclusions Subretinal lymphomatous infiltrates respond to systemic chemotherapy with immunomodulatory maintenance, but dense vitreous infiltrates require therapeutic vitrectomy.
\end{abstract}

\section{Introduction}

Retinal lymphoma (also termed vitreoretinal lymphoma) is usually a high-grade, diffuse large B-cell lymphoma, which usually involves the central nervous system (CNS) $[1,2]$.

Supplementary information The online version of this article (https:// doi.org/10.1038/s41433-019-0539-3) contains supplementary material, which is available to authorized users.

Bertil Damato

bertil.damato@nhs.net

1 Department of Ophthalmology, University of California, San Francisco, USA

2 Nuffield Department of Clinical Neurosciences, University of Oxford, Oxford, UK

3 Department of Hemato-Oncology, University of California, San Francisco, San Francisco, CA, USA
The incidence is increasing [3]. The lymphomatous infiltrates are: retinal, with vascular sheathing; subretinal (i.e., between the retinal pigment epithelium (RPE) and Bruch's membrane); and intra-vitreal. Other features include pseudo-hypopyon, cystoid macular edema, epiretinal membrane formation, and optic neuropathy.

In the absence of overt CNS lymphoma, it is standard practice to perform vitreous biopsy to confirm the diagnosis $[1,4]$. Conventionally, treatment consists of ocular radiotherapy or intravitreal injections of methotrexate, melphalan, or rituximab [5-8]. Treatment of retinal lymphoma without CNS disease is controversial [4, 9, 10]. Several authors have treated retinal lymphoma systemically [11-14]. Some studies suggest that systemic therapy for retinal lymphoma delays CNS disease [15-21]. Other studies indicate no impact on survival [22-24]. In patients with CNS and retinal lymphoma, evidence regarding the impact of ocular treatment on survival is conflicting $[25,26]$. 
At University of California, San Francisco (UCSF), retinal lymphoma is treated systemically, reserving focal ocular therapy for persistent disease. This audit aims to evaluate our approach.

\section{Patients and methods}

Patients were included if they attended our ocular oncology service between June 2013 and March 2018. They were excluded if mostly treated elsewhere or before 2012, when the UCSF electronic medical records system was launched, or if they presented with terminal illness or near the study close.

Ocular examination involved visual acuity measurement with a Snellen or LogMAR chart, tonometry, slit-lamp examination of the anterior chamber and vitreous, binocular indirect ophthalmoscopy, color photography, fundus autofluorescence imaging, optical coherence tomography and, rarely, fluorescein angiography. Vitreous biopsy was performed using the sutureless, three-port, 25- or 27-gauge Constellation system (Alcon Laboratories, Fort Worth, TX, USA) first under air infusion, to obtain a large sample of undiluted vitreous for cytology and flow cytometry before continuing the vitrectomy with balanced salt solution infusion, to collect specimens for analysis for gene rearrangements and, more recently, infectious agents using metagenomics deep sequencing [27]. Laser endophotocoagulation, silicone oil injection, gas tamponade, and combined phacoemulsification were not performed.

Patients with confirmed retinal lymphoma were referred to the haemato-oncology unit for further management. Investigations included baseline $\mathrm{CT}$ of chest, abdomen and pelvis; magnetic resonance imaging of the brain; bone marrow biopsy; lumbar puncture; and serology for hepatitis $\mathrm{B}$ and $\mathrm{C}$ and the human immunodeficiency virus. Systemic therapy usually involved: (a) induction with methotrexate, temozolomide and rituximab; (b) consolidation with etoposide and/or cytarabine; and (c) maintenance with an immunomodulatory agent (i.e., lenalidomide or CC-122). CNS recurrence was treated with methotrexate. Wholebrain radiotherapy was administered only as a last resort with a dose of 36-46 Gy. Focal radiotherapy for ocular or localized CNS disease consisted of intensity-modulated radiotherapy or gamma knife radiosurgery, delivering 30.6 Gy or 15 Gy, respectively.

Clinical findings were documented using the UCSF electronic medical records (APeX, EPIC Systems, Verona, WI, USA).

The date of retinal lymphoma diagnosis was defined as the date when we confirmed or diagnosed this disease. Vitreous infiltration was categorized according to number of cells per $1 \mathrm{~mm}$ beam and/or fundus obscuration on color photography as: (a) absent (no cells); (b) minimal (1-10 cells); (c) mild (11-30 cells and/or obscuration of retinal capillaries); (d) moderate (31-100 cells and/or obscuration of small retinal vessels), and (e) severe (> 100 cells and/or obscuration of large retinal vessels and/or optic disc).

Subretinal infiltrates were categorized as: (a) absent; (b) minimal if subtle and flat ('flecks'); (c) mild if small and raised ('drusenoid'); (d) moderate if confluent infiltrates/ small tumors; and (e) severe if large tumors were present (Fig. 1).

The ocular disease was classified as: (a) purely retinal, without visible vitreous infiltrates, (b) vitreo-retinal; and (c) purely vitreal, without discernible retinal infiltrates.

Statistical analysis was performed with SPSS (IBM Corp., Armonk, NY, USA); and Stata (StataCorp, College Station, TX, USA). We followed the Tenets of the Declaration of Helsinki. This being an audit, this study was exempted by the UCSF Institutional Review Board (IRB\#: 17-24094; Ref. \#: 207748).

\section{Results}

The cohort comprised eight women and two men (Table 1). The median age at ocular lymphoma diagnosis was 58.6 years (range, 38.4-73.0). Ocular symptoms preceded the diagnosis of retinal lymphoma by a median of 6.9 months (range, 0-24.1).

Overt disease was initially bilateral in six patients and unilateral in four, becoming bilateral in two of these. In the 18 affected eyes, vitreous infiltrates were initially absent $(N=2)$, minimal $(N=4)$, mild $(N=7)$, moderate $(N=3)$, and severe $(N=2)$, whereas subretinal infiltrates were absent $(N=6)$, minimal $(N=2)$, mild $(N=3)$, moderate $(N=5)$, severe $(N=1)$, and obscured by vitreous infiltrates $(N=1)$. The initial visual acuity in the 18 affected eyes was: $20 / 20-20 / 25(N=8) ; 20 / 32-20 / 63(N=7) ; 20 / 200$ $(N=1)$, because of rhegmatogenous retinal detachment after vitreous biopsy elsewhere (Case 3); and Count Fingers $(N=2)$, because of fibrosed sub-foveal lymphomatous deposits in Case 4 and dense vitreous infiltrates in Case 7.

Cases 2, 4, 7, and 9 had a history of CNS lymphoma, diagnosed a median of 14.3 months previously (range, 10.4-30.6) (Table 2). In Case 3, the ocular and CNS lymphoma were diagnosed concurrently although this patient had previously undergone unsuccessful biopsy for vitreous infiltrates elsewhere.

The median ophthalmic follow-up was 37.0 months (range, 8.1-56.2). All patients received systemic therapy. Induction therapy included methotrexate, rituximab and temozolomide in all patients, except for Case 9 in whom temozolomide was omitted. Case 2 received $\mathrm{CHOP}$ (cyclophosphamide, doxorubicin, vincristine, prednisolone) 
Fig. 1 Fundus photographs showing grading of subretinal infiltrates: (1) minimal, subtle flecks; (2) mild, prominent flecks; (3) moderate, confluent flecks/small tumors; and (4) severe, large tumors
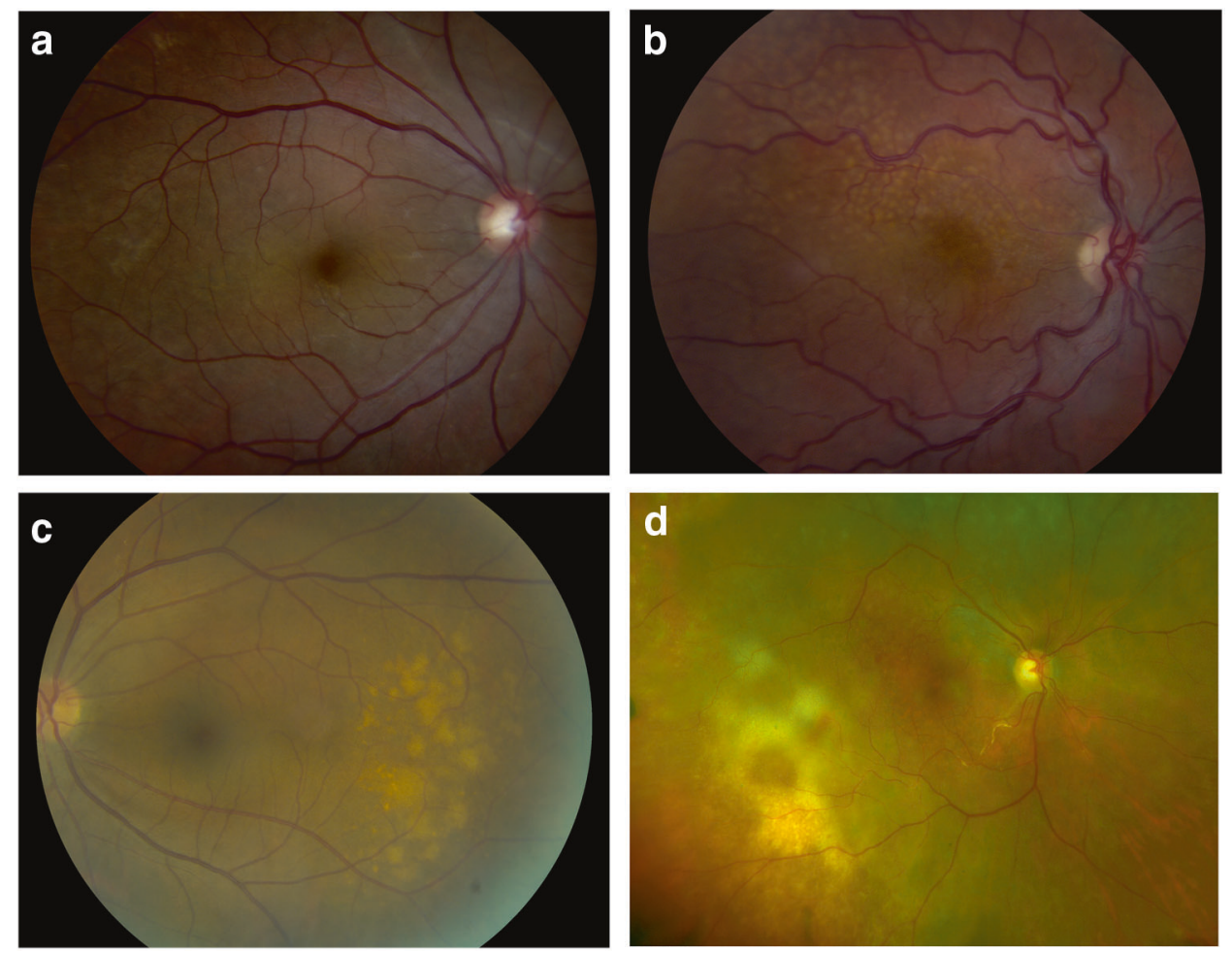

because of a positive bone marrow biopsy at initial assessment. All patients except Case 8 received consolidation therapy with etoposide, with additional cytarabine in Cases 2, 4, 7, and 10. Ten patients received maintenance therapy, consisting of lenalidomide in nine patients and $\mathrm{CC}$ 122 (an investigational immune modulator) in Case 4. Case 2 underwent whole-brain radiotherapy. Diagnostic vitrectomy was performed in 10 eyes and therapeutic vitrectomy was performed in Cases 3 and 10. Cases 2, 5, 6, and 7 received ocular radiotherapy, bilaterally in Cases 2 and 5. Case 7 received intravitreal melphalan injections, twice to the left eye and once to the right eye.

Vitreous opacities persisted despite systemic therapy the right eye of Case 3 and both eyes of Case 7, resolving with therapeutic vitrectomy and intra-vitreal melphalan, respectively. The intraocular disease recurred in the right eye in Case 6 and was treated with radiotherapy. Case 7 developed recurrent vitreous infiltrates resolving with repeated intravitreal melphalan injections. Case 3 developed rhegmatogenous retinal detachment before referral to us and Case 6 developed retinal and choroidal detachment after cataract surgery. Cases 2 and 8 showed lymphomatous vascular sheathing. Seven eyes of five patients developed epiretinal membranes, following rhegmatogenous retinal detachment in Case 3. Case 8 developed bilateral cystoid macular edema. At their first assessment at our clinic, Cases 2 and 6 had bilateral lens opacities and 4 patients were pseudophakic, unilaterally (Cases 3 and 9) or bilaterally (Cases 5 and 8). Cases 3, 6, and 9 underwent uncomplicated phacoemulsification with intraocular lens implantation during the study.

At the study close, vitreous infiltrates were absent $(N=$ $7)$, minimal $(N=9)$, mild $(N=1)$, and moderate $(N=1)$, whereas subretinal infiltrates were absent $(N=7)$, minimal $(N=10)$, and mild $(N=1)$.

The latest visual acuity was: $20 / 20-20 / 25$ ( $N=9$ eyes); 20/32-20/63 $(N=6)$; Count Fingers $(N=2)$, because of sub-foveal scars; and No Light Perception $(N=1)$. Compared with the initial visual acuity, the latest visual acuity was: improved in two eyes, because of resolution of vitreous infiltrates in Case 7 and healing of a macular hole in Case 3; and worse in two eyes, because of sub-foveal scarring in Case 8 and cataract surgery complications in Case 6 (Fig. 2).

CNS disease developed after the ocular disease in Cases 5 and 10, 12.7 and 4.2 months respectively. Follow-up from the date of diagnosis of CNS lymphoma to the latest assessment before the study close had a median of 48.2 months (range, 19.1-55.4). All patients were alive at the study close, with complete remission of their CNS disease, although Case 2 had short-term memory loss, probably caused by whole brain radiotherapy.

\section{Discussion}

The main finding of this study is that subretinal lymphoma deposits responded to systemic induction, consolidation and 


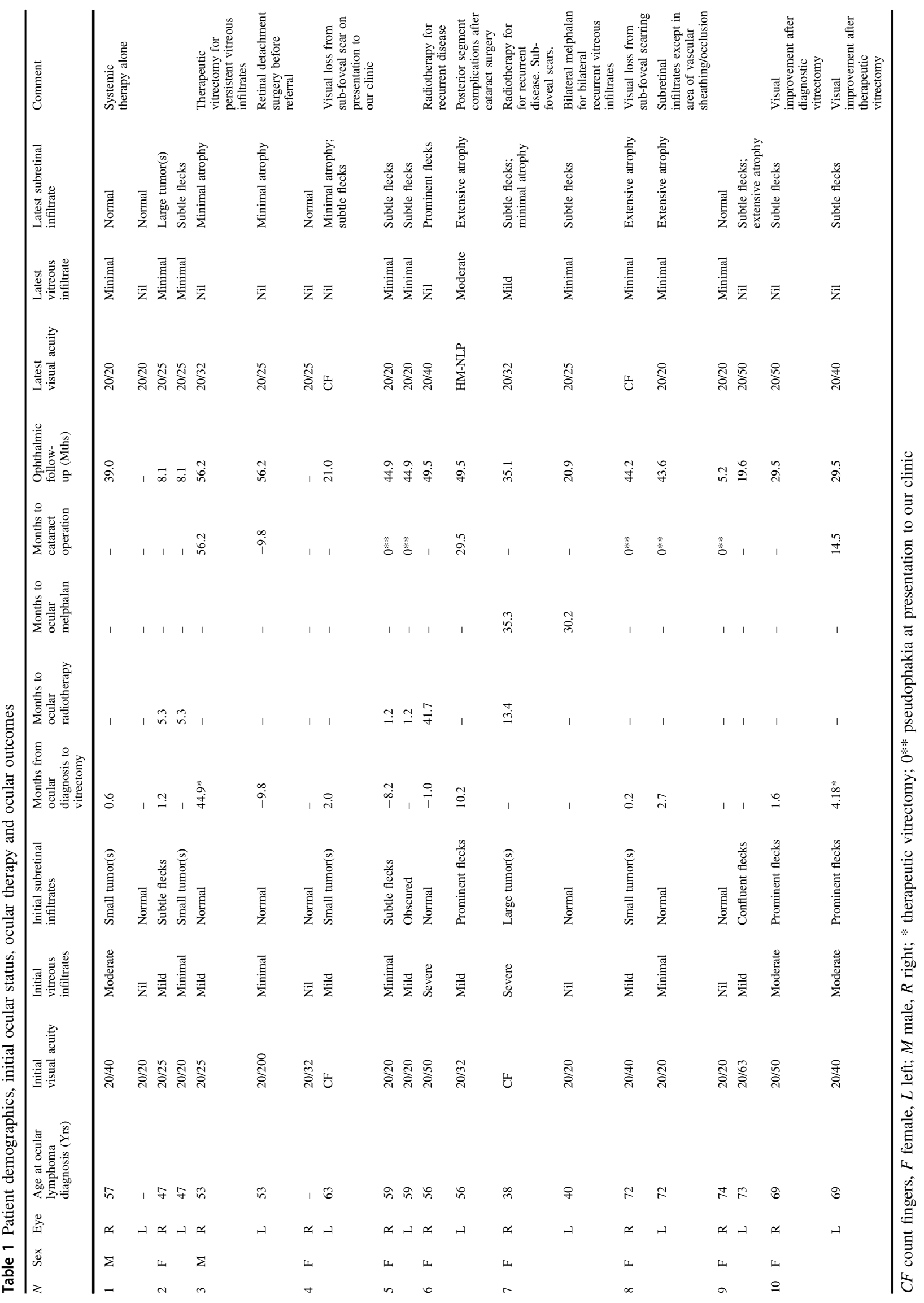




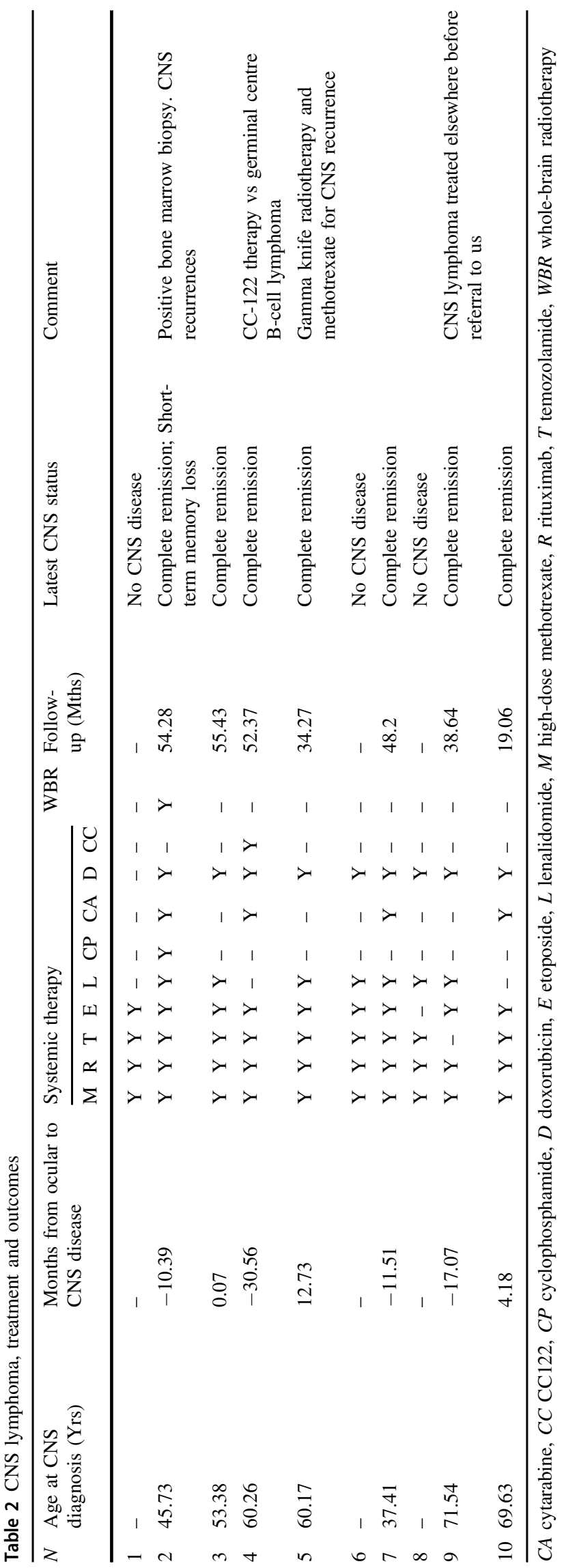

maintenance therapy, which usually included an immune modulator (i.e., lenalidomide or CC-122). Vitreous infiltrates were more resistant unless vitrectomy had been performed. All patients were alive at the study close, with ocular tumor control in all patients, except for one patient. Preservation of vision to pre-treatment levels was good except in an eye that developed sub-foveal lymphomatous deposits and an eye with complications following cataract surgery. Fundus autofluorescence imaging revealed lesions that were not readily apparent with color photography, also allowing differentiation between hyper-autofluorescent subretinal tumor deposits and hypo-autofluorescent atrophic RPE scars.

Strengths of this study are the use of therapeutic vitrectomy and immune modulation with lenalidomide or CC-122. To our knowledge, this is the first cohort study reporting on these methods, which have hitherto been mentioned only in rare case reports. The main weakness of this study is the small number of patients; however, this limited number enabled in-depth analysis and reporting that would not otherwise have been possible. Other weaknesses include subjectivity in the quantification of vitreous cells by slit-lamp examination and inconsistent grading of vitreous opacity because of the use of different cameras during the study.

Our systemic therapy was based on the hypothesis that retinal and CNS lymphomas arise from an occult focus in the bone marrow or elsewhere. Case 8 provides some circumstantial evidence for such systemic spread, because of a paucity of subretinal lymphomatous deposits in a zone supplied by an occluded retinal arteriole [28].

Detailed analysis of systemic drug-induced morbidity is beyond the scope of this study. Such morbidity is discussed in a previous article from our center [29]. Although none of our patients had died by the study close, further clinical trials are needed to determine whether our approach prolongs life.

Most of the affected eyes in our series showed regression of subretinal infiltrates with systemic therapy alone, as has been reported previously, not only with chemotherapy, but also with lenalidomide [30]. Fundus autofluorescence imaging indicates that, as happens with vitrelliform dystrophy, RPE atrophy tended to develop when subretinal tumor deposits regressed, as observed in Case 8 [28]. In some eyes, subtle subretinal infiltrates, which we termed "flecks", persisted, suggesting that the disease was suppressed but not fully eradicated. This hypothesis is supported by Case 5, whose retinal and CNS lymphoma recurred after the close of the study when the lenalidomide was stopped, when she underwent back surgery (laminectomy).

Vitreous infiltrates were more resistant to systemic therapy unless the eye had been vitrectomized. We have therefore started performing therapeutic vitrectomy for vitreous infiltrates not responding to systemic therapy [31]. 
Fig. 2 Initial and final visual acuity (Blue $=$ left eye; Red $=$ right eye)

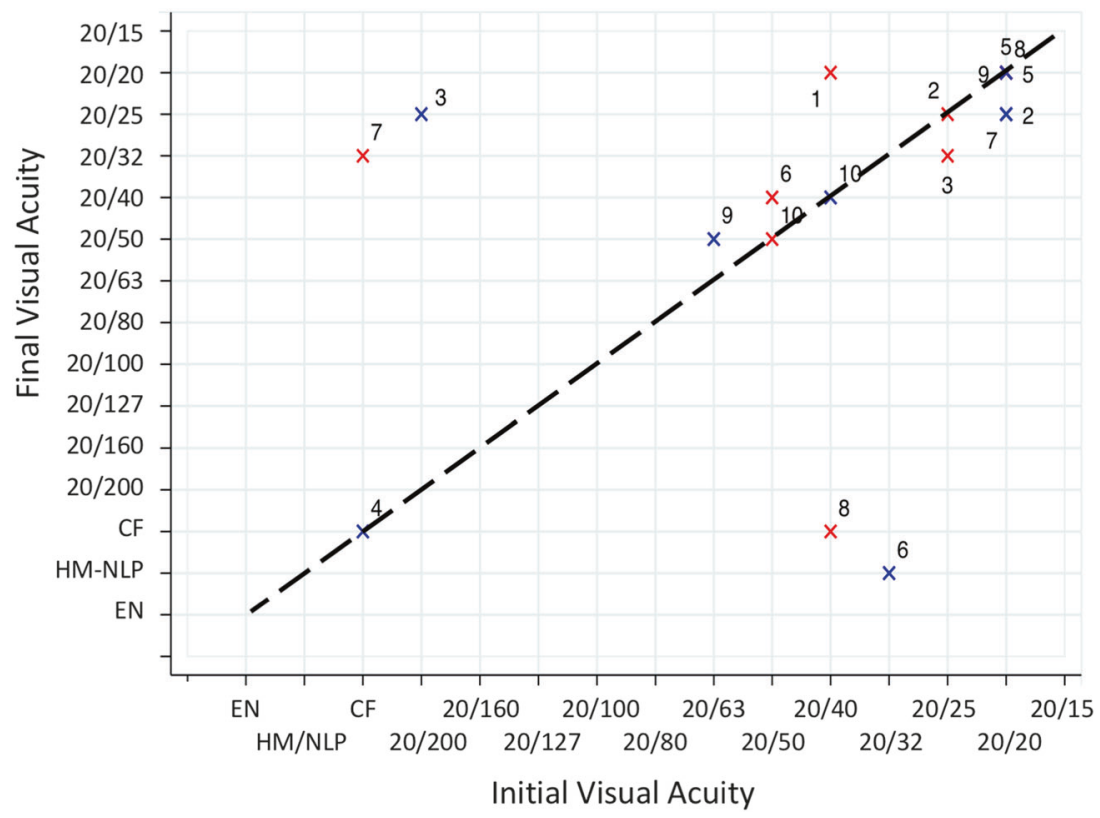

Venkatesh et al. [32] report a case with similar resolution of vitreous infiltrates after vitrectomy. We also gained the impression that subretinal infiltrates were less apparent after vitrectomy. Pakdel et al. [33] report a case with resolution of subretinal lymphoma deposits after vitrectomy. We hypothesize that lymphoma cells spread both directly and/or after proliferating in the vitreous cavity from the retina through the RPE to the subretinal space, where they are trapped by Bruch's membrane. We have seen histology showing retinoblastoma cells accumulating at Bruch's membrane after passing through intact RPE. We also hypothesise that vitrectomy reduces lymphoma cell proliferation in the vitreous, so that fewer of these cells percolate through the RPE and so that they can be cleared from the subretinal space before tumors form.

In Case 4, early vitrectomy would perhaps have prevented sub-foveal accumulation of lymphoma cells and loss of central vision. Case 8 also developed macular atrophy but this was apparently caused by severe cystoid edema.

If lymphoma cell distribution within the eye influences therapeutic response, this would indicate scope for categorizing retinal lymphoma into the severity of intravitreal and subretinal infiltrates, as suggested by Hashida et al. [17]. They found that the distribution of lymphoma within the eye did not correlate with CNS disease, which is not surprising as we have seen patients with predominantly vitreal infiltrates in one eye and mostly retinal infiltrates in the other.

We avoided intravitreal methotrexate and discontinued radiotherapy because of reported toxicity with such treatments $[34,35]$. The good visual outcomes following systemic therapy in our patients support our approach. We treated one patient with intravitreal melphalan; [8] however, when we observed recurrent disease, we scheduled the patient for vitrectomy, which was performed in the left eye after the study close, with resolution of the vitreous infiltrates.

The female preponderance that occurred in this study has been noted previously in some but not all studies $[2,17]$.

Randomized trials are needed to test the following hypotheses: (1) systemic therapy with immunotherapy prolongs life in patients with retinal lymphoma; (2) in patients with unilateral retinal lymphoma, systemic therapy prevents disease in the fellow eye; (3) in patients with significant vitreous lymphomatous infiltrates therapeutic vitrectomy enhances the effect of systemic therapy; and (4) systemic therapy with immunotherapy is superior to ocular radiotherapy or intravitreal chemotherapy in achieving local control of retinal lymphoma, also causing less ocular morbidity.

\section{Conclusions}

We continue to treat retinal lymphoma systemically, performing vitrectomy for eyes with dense vitreous infiltrates, administering ocular therapy only if these fail to achieve local tumor control. Multi-centre studies are needed to evaluate our approach. These would require disease categorization according to the intraocular distribution of lymphomatous infiltrates.

\section{Summary}

\section{What was known before}

- Retinal lymphoma causes significant ocular morbidity and visual loss. 
- Conventional treatment consists of intra-ocular chemotherapy and/or ocular radiotherapy.

- Survival probability is poor because of fatal CNS lymphoma.

\section{What this study adds}

- Retinal lymphoma can be treated with systemic chemotherapy and immunomodulation.

- Vitreous lymphomatous infiltrates are more responsive to systemic therapy if vitrectomy is performed.

Acknowledgements We are grateful to Michele Bloomer, John Gonzales, Melike Pekmezci and Jay Stewart for contributing to patient care. We also wish to acknowledge research support to Dr. Rubenstein from the Leukemia and Lymphoma Society and from the National Cancer Institute NIH R01CA139-83-01A1.FundingThis work was made possible in part, by NIH-NEI EY002162-Core Grant for Vision Research and by the Research to Prevent Blindness Unrestricted Grant.

\section{Compliance with ethical standards}

Conflict of interest JLR receives research funding from Genentech and Celgene.

Publisher's note: Springer Nature remains neutral with regard to jurisdictional claims in published maps and institutional affiliations.

\section{References}

1. Araujo I, Coupland SE. Primary vitreoretinal lymphoma-a review. Asia Pac J Ophthalmol (Philos). 2017;6:283-9.

2. Cassoux N, Merle-Beral H, Leblond V, Bodaghi B, Milea D, Gerber S, et al. Ocular and central nervous system lymphoma: clinical features and diagnosis. Ocul Immunol Inflamm. 2000;8:243-50.

3. Levasseur SD, Wittenberg LA, White VA. Vitreoretinal lymphoma: a 20-year review of incidence, clinical and cytologic features, treatment, and outcomes. JAMA Ophthalmol. 2013;131:50-5.

4. Fend F, Ferreri AJ, Coupland SE. How we diagnose and treat vitreoretinal lymphoma. Br J Haematol. 2016;173:680-92.

5. Frenkel S, Hendler K, Siegal T, Shalom E, Pe'er J. Intravitreal methotrexate for treating vitreoretinal lymphoma: 10 years of experience. Br J Ophthalmol. 2008;92:383-8.

6. Berenbom A, Davila RM, Lin HS, Harbour JW. Treatment outcomes for primary intraocular lymphoma: implications for external beam radiotherapy. Eye. 2007;21:1198-201.

7. Larkin KL, Saboo US, Comer GM, Forooghian F, Mackensen F, Merrill $\mathrm{P}$, et al. Use of intravitreal rituximab for treatment of vitreoretinal lymphoma. Br J Ophthalmol. 2014;98:99-103.

8. Shields CL, Sioufi K, Mashayekhi A, Shields JA. Intravitreal melphalan for treatment of primary vitreoretinal lymphoma: a new indication for an old drug. JAMA Ophthalmol. 2017;135:815-8.

9. Chan CC, Rubenstein JL, Coupland SE, Davis JL, Harbour JW, Johnston PB, et al. Primary vitreoretinal lymphoma: a report from an International Primary Central Nervous System Lymphoma Collaborative Group symposium. Oncologist. 2011;16:1589-99.
10. Witmer MT. Primary vitreoretinal lymphoma: management of isolated ocular disease. Cancer Control. 2016;23:110-6.

11. Jahnke K, Bechrakis NE, Coupland SE, Schmittel A, Foerster MH, Fischer L, et al. Treatment of primary intraocular lymphoma with oral trofosfamide: report of two cases and review of the literature. Graefe's Arch Clin Exp Ophthalmol. 2004;242:771-6.

12. Soussain C, Merle-Beral H, Reux I, Sutton L, Fardeau C, Gerber S, et al. A single-center study of 11 patients with intraocular lymphoma treated with conventional chemotherapy followed by high-dose chemotherapy and autologous bone marrow transplantation in 5 cases. Leuk lymphoma. 1996;23:339-45.

13. Nguyen DT, Houillier C, Choquet S, Cassoux N, Soussain C, Le Cossec C, et al. Primary oculocerebral lymphoma: MTX polychemotherapy alone on intraocular disease control. Ophthalmology. 2016;123:2047-50.

14. Batchelor TT, Kolak G, Ciordia R, Foster CS, Henson JW. Highdose methotrexate for intraocular lymphoma. Clin Cancer Res. 2003;9:711-5.

15. Hormigo A, Abrey L, Heinemann MH, DeAngelis LM. Ocular presentation of primary central nervous system lymphoma: diagnosis and treatment. Br J Haematol. 2004;126:202-8.

16. Valluri S, Moorthy RS, Khan A, Rao NA. Combination treatment of intraocular lymphoma. Retina. 1995;15:125-9.

17. Hashida N, Nakai K, Saitoh N, Nishida K. Association between ocular findings and preventive therapy with onset of central nervous system involvement in patients with primary vitreoretinal lymphoma. Graefe's Arch Clin Exp Ophthalmol. 2014;252:687-93.

18. Jahnke K, Korfel A, Komm J, Bechrakis NE, Stein H, Thiel E, et al. Intraocular lymphoma 2000-5: results of a retrospective multicentre trial. Graefe's Arch Clin Exp Ophthalmol. 2006;244:663-9.

19. Kim MM, Dabaja BS, Medeiros J, Kim S, Allen P, ChevezBarrios P, et al. Survival outcomes of primary intraocular lymphoma: a single-institution experience. Am J Clin Oncol. 2016;39:109-13.

20. Klimova A, Heissigerova J, Rihova E, Brichova M, Pytlik R, Spicka I et al. Combined treatment of primary vitreoretinal lymphomas significantly prolongs the time to first relapse. $\mathrm{Br} \mathrm{J}$ Ophthalmol 2018;102:1579-85.

21. Ma WL, Hou HA, Hsu YJ, Chen YK, Tang JL, Tsay W, et al. Clinical outcomes of primary intraocular lymphoma patients treated with front-line systemic high-dose methotrexate and intravitreal methotrexate injection. Ann Hematol. 2016;95:593-601.

22. Riemens A, Bromberg J, Touitou V, Sobolewska B, Missotten T, Baarsma S, et al. Treatment strategies in primary vitreoretinal lymphoma: a 17-center European collaborative study. JAMA Ophthalmol. 2015;133:191-7.

23. Grimm SA, Pulido JS, Jahnke K, Schiff D, Hall AJ, Shenkier TN, et al. Primary intraocular lymphoma: an International Primary Central Nervous System Lymphoma Collaborative Group Report. Ann Oncol. 2007;18:1851-5.

24. Cho BJ, Kim DY, Park UC, Lee JY, Yoon YH, Yu HG. Clinical features and treatment outcomes of vitreoretinal lymphoma according to its association with CNS lymphoma. Ocul Immunol Inflamm 2018;26:1-7.

25. Grimm SA, McCannel CA, Omuro AM, Ferreri AJ, Blay JY, Neuwelt EA, et al. Primary CNS lymphoma with intraocular involvement: International PCNSL Collaborative Group Report. Neurology. 2008;71:1355-60.

26. Ferreri AJ, Blay JY, Reni M, Pasini F, Gubkin A, Tirelli U, et al. Relevance of intraocular involvement in the management of 
primary central nervous system lymphomas. Ann Oncol. 2002;13:531-8.

27. Gonzales J, Doan T, Shantha JG, Bloomer M, Wilson MR, DeRisi JL, et al. Metagenomic deep sequencing of aqueous fluid detects intraocular lymphomas. $\mathrm{Br} \mathrm{J}$ Ophthalmol. 2018;102:6-8.

28. Damato BE, Bever GJ, Afshar AR, Rubenstein JL. Insights from a Case of Vitreoretinal Lymphoma. Ocul Oncol Pathol. 2019:13-9. https://doi.org/10.1159/000487949.

29. Carnevale J, Rubenstein JL. The challenge of primary central nervous system lymphoma. Hematol Oncol Clin North Am. 2016;30:1293-316.

30. Rubenstein JL, Treseler PA, Stewart PJ. Regression of refractory intraocular large B-cell lymphoma with lenalidomide monotherapy. J Clin Oncol. 2011;29:e595-7.

31. Bever GJ, Kim DJ, Afshar AR, Rubenstein JL, Damato BE. Therapeutic vitrectomy as an adjunct treatment to systemic chemotherapy for intraocular lymphoma. Retin Cases Brief Rep 2017. https://doi.org/10.1097/ICB.0000000000000668.

32. Venkatesh P, Gogia V, Khanduja S, Gupta S, Kumar L, Garg S. Therapeutic vitrectomy for vitreal recurrence of intraocular lymphoma resistant to intravitreal methotrexate post systemic chemotherapy. J Cancer Res Ther. 2015;11:668.

33. Pakdel A, Mammo Z, Hollands H, Forooghian F. Regression of subretinal lymphoma after diagnostic vitrectomy. JAMA Ophthalmol. 2017;135:503-5.

34. Hoffman PM, McKelvie P, Hall AJ, Stawell RJ, Santamaria JD. Intraocular lymphoma: a series of 14 patients with clinicopathological features and treatment outcomes. Eye. 2003;17:513-21.

35. Smith JR, Rosenbaum JT, Wilson DJ, Doolittle ND, Siegal T, Neuwelt EA, et al. Role of intravitreal methotrexate in the management of primary central nervous system lymphoma with ocular involvement. Ophthalmology. 2002;109:1709-16. 